\title{
Prognostic value of neutrophil-to-lymphocyte ratio in locally advanced non-small cell lung cancer treated with concurrent chemoradiotherapy
}

\author{
Eun Young Park', Yeon-Sil Kim ${ }^{1}$, Kyu Hye Choi ${ }^{1}$, Jin Ho Song ${ }^{1}$, Hyo Chun Lee ${ }^{1}$, \\ Sook-Hee Hong' ${ }^{2}$ Jin-Hyoung Kang ${ }^{2}$
}

'Department of Radiation Oncology, Seoul St. Mary's Hospital, College of Medicine, The Catholic University of Korea, Seoul, Korea ${ }^{2}$ Department of Medical Oncology, Seoul St. Mary's Hospital, College of Medicine, The Catholic University of Korea, Seoul, Korea

Purpose: This study aimed to investigate neutrophil-to-lymphocyte ratio (NLR) and platelet-to-lymphocyte ratio (PLR) as prognostic factors in patients with locally advanced non-small cell lung cancer (NSCLC) who received concurrent chemoradiotherapy (CCRT).

Materials and Methods: We retrospectively analyzed 66 patients with locally advanced NSCLC treated with definitive CCRT. Among these patients, 95\% received paclitaxel/carboplatin or docetaxel/cisplatin. The median radiation dose was 66 Gy in 33 fractions. The NLR and PLR before/after CCRT were evaluated. The maximally selected log-rank test was used to obtain the cutoff values related to the overall survival (OS).

Results: Patients with high post-CCRT NLR ( $>3.12)$ showed worse OS, locoregional progression-free survival (LRPFS), and distant metastasis-free survival (DMFS) than those with low NLR (2-year OS: $25.8 \%$ vs. 68.2\%, $p<0.001 ; 2$-year LRPFS: $12.9 \%$ vs. $33.8 \%$, $p=0.010 ; 2$-year DMFS: $22.6 \%$ vs. $38.2 \%, p=0.030)$. Patients with high post-CCRT PLR ( $>141)$ showed worse OS and LRPFS than those with low PLR (2-year OS: $37.5 \%$ vs. $71.1 \%, p=0.004 ; 2$-year LRPFS: $16.5 \%$ vs. $40.3 \%, p=0.040$ ). Patients with high NLR change ( $>1.61$ ) showed worse OS and LRPFS than those with low NLR change (2-year OS: $26.0 \%$ vs. 59.0\%, $p<0.001 ; 2$-year LRPFS: $6.8 \%$ vs. $31.8 \%, p=0.004)$. The planning target volume (hazard ration $[H R]=2.05, p=0.028)$ and NLR change $(H R=3.17, p=0.025)$ were the significant factors for $\mathrm{OS}$ in the multivariate analysis.

Conclusion: NLR change after CCRT was associated with poor prognosis of survival in patients with locally advanced NSCLC. An elevated NLR after CCRT might be an indicator of an increased treatment failure risk.

Keywords: Neutrophil-to-lymphocyte ratio, Platelet-to-lymphocyte ratio, Non-small cell lung cancer, Concurrent chemoradiotherapy

\section{Introduction}

Lung cancer is the most common cancer and a leading cause of cancer death globally [1]. In about 85\% cases, lung cancer is diagnosed as non-small cell lung cancer (NSCLC) [2]. In NSCLC, $20 \%-25 \%$ of patients are diagnosed with

Received 22 April 2019, Revised 20 June 2019, Accepted 08 July 2019.

Correspondence: Yeon-Sil Kim, Department of Radiation Oncology, Seoul St. Mary's Hospital, College of Medicine, The Catholic University of Korea, 222, Banpo-daero, Seocho-gu, Seoul 06591, Korea. Tel: +82-2-2258-1526, Fax: +82-2-592-1532, E-mail: yeonkim7@catholic.ac.kr (http://orcid.org/0000-0003-1291-0821)

(c) This is an Open Access article distributed under the terms of the Creative Commons Attribution Non-Commercial License (http://creativecommons.org/ licenses/by-nc/4.0/) which permits unrestricted non-commercial use, distribution, and reproduction in any medium, provided the original work is properly cited.

www.e-roj.org 
locally advanced disease (stages IIIA and IIIB according to the American Joint Committee on Cancer [AJCC] 7th edition) [3]. Patients with inoperable locally advanced NSCLC have a poor prognosis, with a 5-year survival rate of 15\%-25\% [4].

For locally advanced NSCLC, multimodality treatment is used. Patients with resectable stage IIIA NSCLC undergo surgery or neoadjuvant chemotherapy/chemoradiotherapy. The standard treatment of unresectable locally advanced NSCLC is definitive concurrent chemoradiotherapy (CCRT) [5]. Despite multimodality treatment, local and distant failure is high in unresectable locally advanced NSCLC. Thus, there is a need to determine the prognostic and predictive factors in locally advanced NSCLC to improve treatment strategy.

The prognostic and predictive factors of NSCLC are known as disease stage, performance status, sex, age, histology, tumor size, and mediastinal infiltration [2]. Recently, routinely assessed biological variables, such as neutrophil-tolymphocyte ratio (NLR), platelet-to-lymphocyte ratio (PLR), and leukocytosis have been suggested as the prognostic factors [3,6-13]. Templeton et al. conducted a meta-analysis of 100 studies with a total of 40,599 patients and they demonstrated that a high NLR is associated with poor overall survival (OS) in many solid tumors [6]. Another meta-analysis of 20 studies with 12,754 patients showed that high PLR is associated with poor OS in various cancers [7]. Several studies have shown that NLR and PLR are associated with the prognosis in patients with stage I-IV NSCLC [3,6,10-12]. However, previous studies had heterogeneity in stage and treatment modalities.

This study aimed to evaluate the prognostic and predictive value of NLR and PLR in patients with locally advanced NSCLC with CCRT as first-line treatment.

\section{Materials and Methods}

\section{Patients}

We retrospectively analyzed 66 patients with locally advanced NSCLC treated with CCRT between 2008 and 2017 at our hospital. The inclusion criteria were as follows: (1) new diagnosis of stage IIIA or IIIB NSCLC according to the 7th edition of the TNM classification of the AJCC, (2) histologically confirmed NSCLC, (3) Eastern Cooperative Oncology Group (ECOG) performance score of $0-2$, and (4) lymphocyte and neutrophil counts performed before and after CCRT. The exclusion criteria were as follows: (1) received induction chemotherapy; (2) non-completion of the planned treatment and treatment of $\leq 50 \mathrm{~Gy}$, (3) history of hematologic malignancies or chemotherapy for other diseases, and (4) evidence of acute infection. A total of 66 patients met the inclusion/exclusion criteria and received definitive CCRT as the first-line treatment. This study was approved by the Institutional Review Board of Seoul St. Mary's Hospital (No. KC19RESE0254).

\section{Chemotherapy}

CCRT consisted of weekly chemotherapy using paclitaxel/ carboplatin (PC), docetaxel/cisplatin (DP), docetaxel/carboplatin, and etoposide/cisplatin. PC was administered to 37 patients (56.1\%), and DP was administered to 26 patients (39.4\%). PC chemotherapy was performed with carboplatin (area under the curve $[A \cup C]=2)$ and paclitaxel $\left(50 \mathrm{mg} / \mathrm{m}^{2}\right)$ administered on a weekly schedule during CCRT. Docetaxel $20 \mathrm{mg} / \mathrm{m}^{2}$ and cisplatin $20 \mathrm{mg} / \mathrm{m}^{2}$ were administered concomitantly with a weekly schedule.

\section{Radiotherapy}

Radiotherapy was performed with intensity-modulated radiotherapy or three-dimensional conformal radiotherapy. The gross tumor volume (GTV) included both primary lung mass and involved lymph nodes visible on imaging $(\geq 1 \mathrm{~cm}$ on CT and/or PET-CT) or histologically proven nodes by endobronchial ultrasound (EBUS) or endoscopic ultrasound (EUS). The clinical target volume (CTV) was defined as an extension of $5 \mathrm{~mm}$ in all directions of the GTV. The planning target volume (PTV) was generated by expanding an additional margin (generally 5-10 $\mathrm{mm}$ ) to the CTV for the setup error and respiratory movement according to the assessment in four-dimensional CT. Elective nodal irradiation was not allowed. The median radiation dose was $66 \mathrm{~Gy}$ in 33 fractions (range, 52 to $72.6 \mathrm{~Gy}$ ).

\section{NLR and PLR}

The pre-CCRT NLR and PLR were calculated from the nearest complete blood count (CBC) within 1 week before the start of CCRT. The post-CCRT NLR and PLR were calculated 4 weeks after CCRT. When CBC was performed several times after CCRT, it was calculated using the $\mathrm{CBC}$ values nearest to the 4 weeks' time interval from the end of the CCRT. For patients who underwent consolidation chemotherapy after CCRT, post-CCRT NLR and NLR were calculated using CBC before consolidation chemotherapy. NLR change was calculated by dividing the post-CCRT NLR by the pre-CCRT NLR. The PLR change was also calculated by dividing the post-CCRT value by the pre-CCRT value. 


\section{Response and follow-up}

The treatment response was evaluated by Response Evaluation Criteria In Solid Tumors (RECIST) criteria. Locoregional progression was defined as progressive disease in the ipsilateral lung, both mediastinal lymph nodes, and both supraclavicular regions. Distant metastasis was defined as any metastasis outside the chest and contralateral lung.

\section{Statistical analysis}

The maximally selected log-rank test was used to acquire the most significant NLR and PLR level related with OS. Continuous variables were analyzed using the independent t-test or Mann-Whitney $U$ test, and categorical variables were analyzed using the chi-square test. We primarily evaluated the OS difference according to NLR and PLR cutoff level and also observed the difference in locoregional progression-free survival (LRPFS) and distant metastasis-free survival (DMFS). The OS, LRPFS, and DMFS were calculated from the first day of CCRT to the date of death, locoregional recurrence, and distant metastasis or most recent follow-up visit using the KaplanMeier method. Univariate analysis was performed using the log-rank test including all patients, tumor related factors, and hematological parameters. The significant variables on univariate analysis were evaluated on multivariate analysis. Multivariate analysis was performed including the lymphocyterelated hematological parameters to eliminate collinearity between the parameters, using Cox proportional hazard model (method, forward). All tests were two-sided, and p-values of $<0.05$ indicated statistical significance. All statistical analyses were performed using R version 3.5.3 (R Development Core Team, Vienna, Austria).

\section{Results}

\section{Patient characteristics}

Patients' characteristics are shown in Table 1. The median age was 64 years (range, 40 to 78 years), and most patients were male (84.8\%). There were 23 patients (34.8\%) with stage IIIA, and 43 patients (65.2\%) with stage IIIB. A total of 33 patients (50.0\%) were diagnosed with squamous cell carcinoma, and 29 patients (43.9\%) were diagnosed with adenocarcinoma. Moreover, 37 patients (56.1\%) received PC chemotherapy, and 26 patients (39.4\%) received DP chemotherapy. Most patients (95.5\%) had ECOG performance status 0-1. The median preCCRT NLR, post-CCRT NLR, and NLR change were 2.7 (range, 1.03 to 16.03 ), 3.0 (range, 0.74 to 44.62 ), and 1.2 (range, 0.05 to 11.02 ), respectively. Furthermore, the median pre-CCRT
PLR, post-CCRT PLR, and PLR change were 145.5 (range, 75 to 453), 172.5 (range, 38 to 898), and 1.1 (range, 0.33 to 7.18), respectively.

\section{Cut-off values of NLR and PLR}

The maximally selected log-rank test was performed to determine optimal cutoff values of NLR and PLR that predict OS. According to the results of the statistics, the optimal cutoff levels were 1.64, 3.12, and 1.61 for the pre-CCRT NLR, post-CCRT NLR, and NLR change. Moreover, the optimal cutoff levels were 115,141 , and 1.67 for the pre-CCRT PLR, post-CCRT $P L R$, and PLR change. The survival analysis was performed by dividing the patients into two groups based on NLR change ( $\leq 1.61$ or $>1.61)$ which is most significant for treatment outcome.

Radiation dose, PTV volume, post-CCRT NLR, post CCRT PLR, and PLR change were statistically different between the two NLR change groups. The high NLR change group had higher radiation dose and larger PTV volume than those in the low $\operatorname{NLR}$ group ( $p=0.035$ and $p=0.024$, respectively). The levels of post-CCRT NLR, post-CCRT PLR, and PLR change were higher in the high NLR change group (all $p<0.001$ ) (Table 1).

\section{OS, LRPFS, and DMFS according to NLR and PLR}

The median follow-up duration was 15.4 months (range, 1.5 to 87.7 months). The 1- and 2-year OS rates were $66.7 \%$ and 48.1\%, respectively. The post-CCRT NLR, PLR and NLR change were significantly associated with OS on univariate analysis. The high post-CCRT NLR group showed significantly worse OS compared to the low NLR group (2-year OS: $25.8 \%$ vs. $68.2 \%$, $p<0.001)$. Moreover, the high post-CCRT PLR had worse OS than the low PLR group (2-year OS: $37.5 \%$ vs. $71.1 \%, p=0.004$ ). The high NLR change group had worse OS than the low NLR change group (2-year OS: $26.0 \%$ vs. $59.0 \%, p<0.001$ ). The Kaplan-Meier survival curves for OS according to NLR and PLR are shown in Fig. 1.

The 1- and 2-year LRPFS rates were $37.0 \%$ and $23.8 \%$, respectively. The post-CCRT NLR, PLR, and NLR change were significantly associated with LRPFS. The high post-CCRT NLR group showed significantly worse LRPFS compared to the low NLR group (2-year LRPFS: 12.9 vs. $33.8 \%, p=0.010$ ). The high post-CCRT PLR group had worse LRPFS than the low PLR group (2-year LRPFS: $16.5 \%$ vs. $40.3 \%, p=0.040$ ). The high NLR change group had worse LRPFS than the low NLR change group (2-year LRPFS: $6.8 \%$ vs. $31.8 \%, p=0.004)$. The KaplanMeier survival curves for LRPFS according to NLR and PLR are shown in Fig. 2. 
Table 1. Patients' characteristics

\begin{tabular}{|c|c|c|c|c|}
\hline \multirow{2}{*}{ Characteristic } & \multirow{2}{*}{ All } & \multicolumn{2}{|c|}{ NLR change } & \multirow{2}{*}{$p$-value } \\
\hline & & $\leq 1.61$ & $>1.61$ & \\
\hline Gender & & & & 0.476 \\
\hline Male & $56(84.8)$ & 36 (81.8) & $20(90.9)$ & \\
\hline Female & $10(15.2)$ & 8 (18.2) & $2(9.1)$ & \\
\hline Age (yr) & & & & 0.066 \\
\hline$\leq 65$ & $36(54.5)$ & $28(63.6)$ & $8(36.4)$ & \\
\hline$>65$ & $30(45.5)$ & $16(36.4)$ & $14(63.6)$ & \\
\hline Stage & & & & 0.927 \\
\hline$\| I A$ & $23(34.8)$ & $16(36.4)$ & 7 (31.8) & \\
\hline IIIB & $43(65.2)$ & 28 (63.6) & $15(68.2)$ & \\
\hline T stage & & & & 0.654 \\
\hline T0-2 & $25(37.9)$ & $18(40.9)$ & 7 (31.8) & \\
\hline T3-4 & $41(62.1)$ & $26(59.1)$ & $15(68.2)$ & \\
\hline N stage & & & & 0.692 \\
\hline No-1 & $6(9.1)$ & $5(11.3)$ & $1(4.5)$ & \\
\hline N2 & $26(39.4)$ & $16(36.4)$ & $10(45.5)$ & \\
\hline N3 & $34(51.5)$ & $23(52.3)$ & $11(50.0)$ & \\
\hline Smoking history & & & & 0.737 \\
\hline No & $12(18.2)$ & $9(20.5)$ & $3(13.6)$ & \\
\hline Yes & $54(81.8)$ & $35(79.5)$ & $19(86.4)$ & \\
\hline ECOG performance status & & & & 0.815 \\
\hline 0 & $28(42.5)$ & $20(45.5)$ & $8(36.4)$ & \\
\hline 1 & $35(53.0)$ & $22(50.0)$ & $13(59.1)$ & \\
\hline 2 & $3(4.5)$ & $2(4.5)$ & $1(4.5)$ & \\
\hline Pathology & & & & 0.915 \\
\hline Squamous cell carcinoma & $33(50.0)$ & $21(47.7)$ & $12(54.6)$ & \\
\hline Adenocarcinoma & $29(43.9)$ & $20(45.5)$ & $9(40.9)$ & \\
\hline Others & $4(6.1)$ & $3(6.8)$ & $1(4.5)$ & \\
\hline Chemotherapy regimen & & & & 0.738 \\
\hline Paclitaxel/carboplatin & $37(56.1)$ & $23(52.3)$ & $14(63.7)$ & \\
\hline Docetaxel/cisplatin & $26(39.4)$ & $19(43.2)$ & 7 (31.8) & \\
\hline Others & $3(4.5)$ & $2(4.5)$ & $1(4.5)$ & \\
\hline Radiation dose (cGy) & $6,600(5,200-7,260)$ & $6,600(6,000-7,260)$ & $6,600(5,200-7,000)$ & 0.035 \\
\hline PTV volume $(\mathrm{mL})$ & $430(119-1,050)$ & $384(119-964)$ & $488(275-1,050)$ & 0.024 \\
\hline Pre-CCRT NLR & $2.7(1.03-16.03)$ & $2.7(1.03-16.03)$ & $2.2(1.09-4.46)$ & 0.176 \\
\hline Pre-CCRT PLR & $145.5(75.0-453.0)$ & $160(75-453)$ & $132.5(85-257)$ & 0.165 \\
\hline Post-CCRT NLR & $3.0(0.74-44.62)$ & $2.4(0.74-7.23)$ & $6.0(2.63-44.62)$ & $<0.001$ \\
\hline Post-CCRT PLR & $172.5(38-898)$ & $148.5(38-379)$ & 265.5 (99-898) & $<0.001$ \\
\hline NLR change & $1.2(0.05-11.02)$ & $0.8(0.05-1.61)$ & $2.5(1.65-11.02)$ & $<0.001$ \\
\hline PLR change & $1.1(0.33-7.18)$ & $0.9(0.33-1.9)$ & $1.9(0.91-7.18)$ & $<0.001$ \\
\hline CRP (mg/dL) & $0.9(0.02-11.51)$ & $0.9(0.02-10.66)$ & $1.1(0.08-11.51)$ & 0.663 \\
\hline $\mathrm{ESR}(\mathrm{mm} / \mathrm{hr})$ & $43(2-120)$ & $40.0(2-120)$ & $47.5(4-120)$ & 0.647 \\
\hline LDH (U/L) & 419 (213-855) & $426(213-855)$ & 396 (282-603) & 0.973 \\
\hline
\end{tabular}

Values are presented as number (\%) or median (range).

ECOG, Eastern Cooperative Oncology Group; PTV, planning target volume; CCRT, concurrent chemoradiotherapy; NLR, neutrophil-to-lymphocyte ratio; PLR, platelet-to-lymphocyte ratio; NLR change, dividing the post-CCRT NLR by the pre-CCRT NLR; PLR change, dividing the post-CCRT PLR by the pre-CCRT PLR; CRP, C-reactive protein; ESR, erythrocyte sedimentation rate; LDH, lactate dehydrogenase. 
A

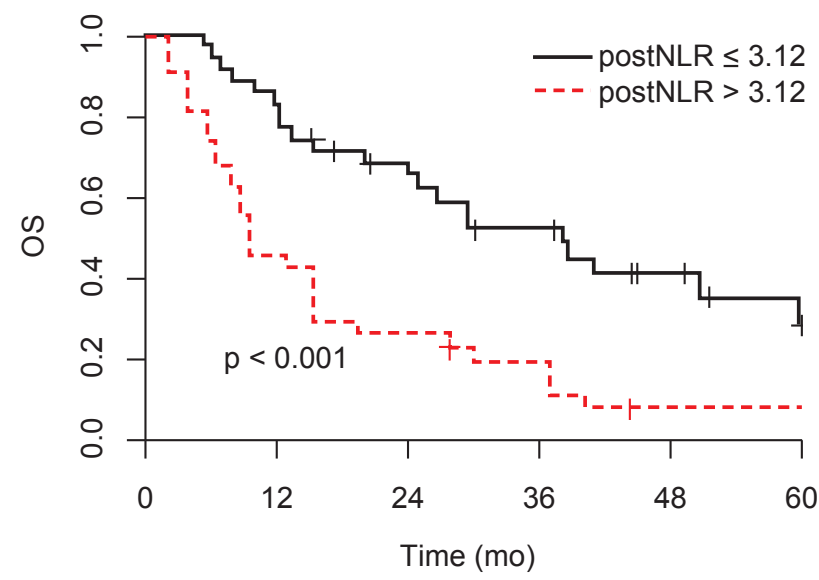

B

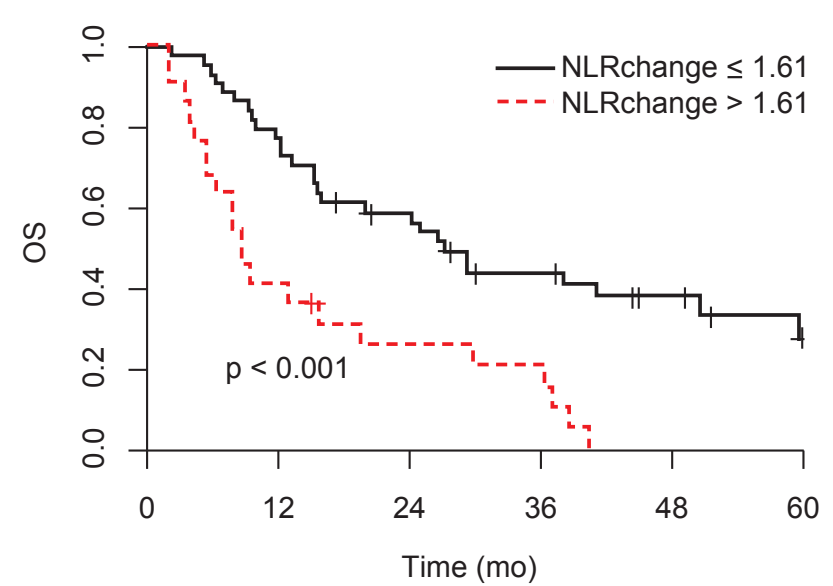

C

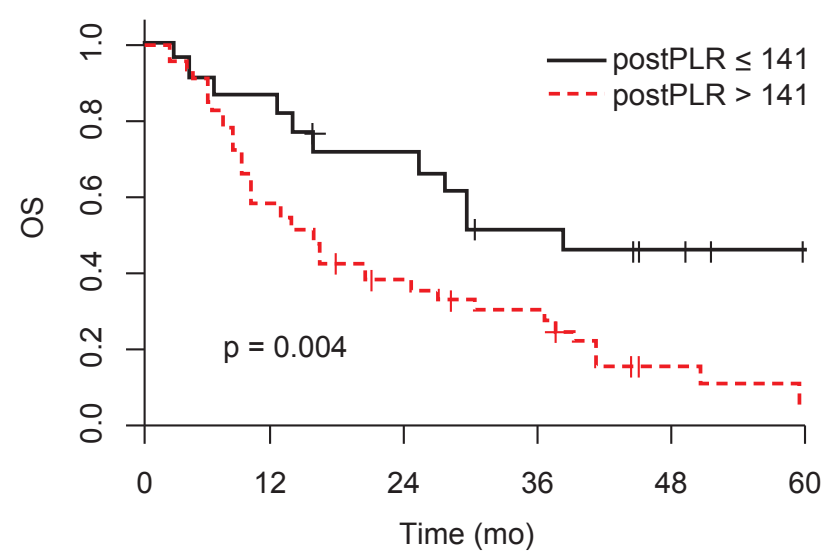

Fig. 1. Overall survival (OS) according to NLR and PLR: (A) post-CCRT NLR, (B) NLR change (post-CCRT NLR / pre-CCRT $N L R$ ), and (C) post-CCRT PLR. NLR, neutrophil-to-lymphocyte ratio; PLR, platelet-to-lymphocyte ratio; CCRT, concurrent chemoradiotherapy.
A

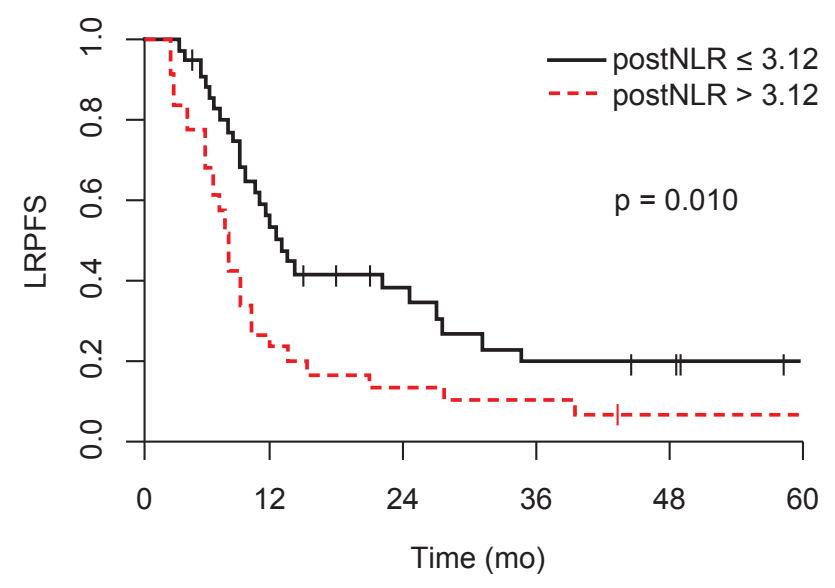

B

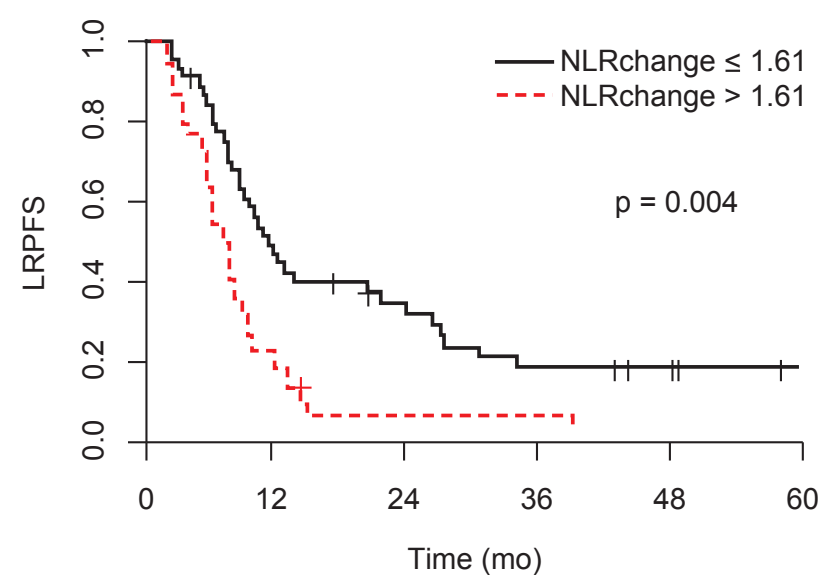

C

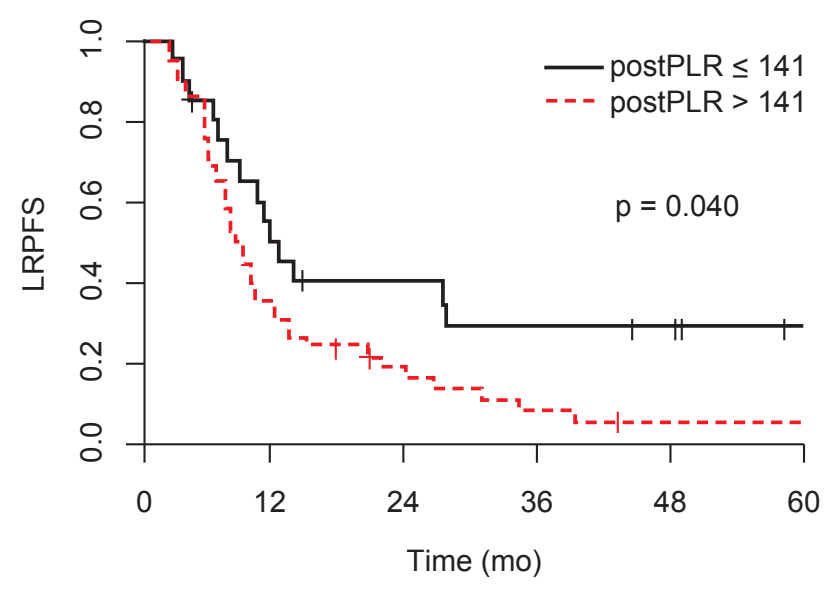

Fig. 2. Locoregional progression-free survival (LRPFS) according to to NLR and PLR: (A) post-CCRT NLR, (B) NLR change (post-CCRT NLR / pre-CCRT NLR), and (C) post-CCRT PLR. NLR, neutrophilto-lymphocyte ratio; PLR, platelet-to-lymphocyte ratio; CCRT, concurrent chemoradiotherapy. 


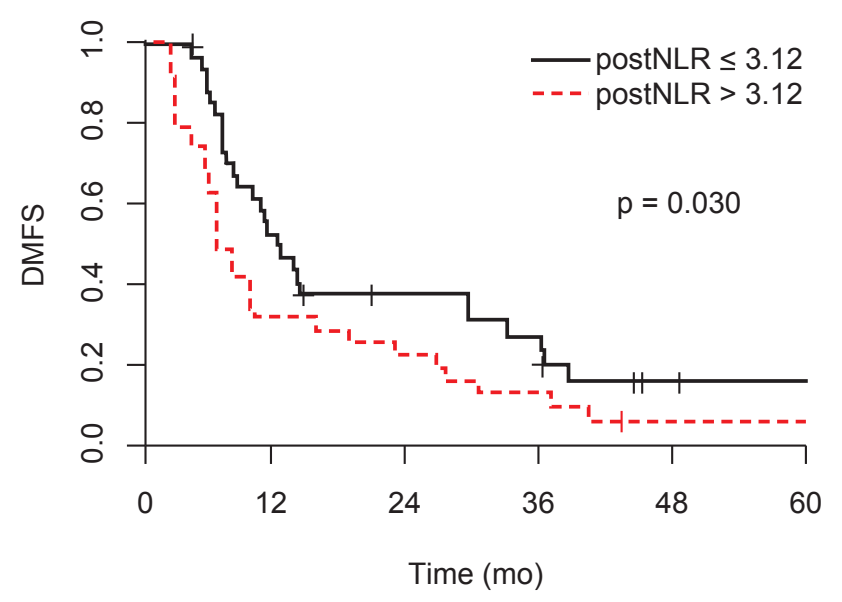

Fig. 3. Distant metastasis-free survival (DMFS) according to post-CCRT neutrophil-to-lymphocyte ratio (NLR).

The 1- and 2-year DMFS rates for all patients were 43.2\% and $30.5 \%$, respectively. The high post-CCRT NLR was significantly associated with worse DMFS. The median DMFS for high post-CCRT NLR group was 6.9 months compared with 12.2 months for low NLR group ( $p=0.030$ ) (Fig. 3).

\section{Prognostic factor}

We performed a univariate analysis to identify the prognostic factor for OS and LRPFS. Univariate analysis showed that PTV volume, post-CCRT NLR, post-CCRT PLR, and NLR change were significantly associated with OS (Table 2). Age, gender, stage, ECOG, pathology, chemotherapy regimen, other inflammatory marker (ESR, CRP, and LDH), pre-CCRT NLR, and PLR were not associated with OS. The PTV volume (hazard ratio $[H R]=2.05$; 95\% confidence interval $[\mathrm{Cl}], 1.08-3.90 ; \mathrm{p}=0.028$ ) and NLR change $(H R=3.17 ; 95 \% C l, 1.16-8.69 ; p=0.025)$ were found as the significant prognostic factors for $0 \mathrm{~S}$ in the multivariate analysis (Table 3).

The post-CCRT NLR, post-CCRT PLR, and NLR change were also significantly associated with LRPFS on univariate analysis (Table 2). In the multivariate analysis for LRPFS, the post-CCRT NLR, post-CCRT PLR, and NLR change did not predict LRPFS (Table 3).

\section{Discussion and Conclusion}

Recent studies have shown that inflammation-related biomarkers were correlated with prognosis in many solid cancers such as colorectal cancer, hepatocellular carcinoma, and NSCLC [3,6-13]. Among inflammation-related biomarkers, NLR and PLR are affordable and easily available markers using
CBC.

The correlation of NLR with survival rate could be explained by tumor-related inflammation and immunity $[3,10,14-16]$. NLR is calculated by dividing neutrophils into lymphocytes, which reflect neutrophilia or lymphopenia. Emerging evidence of animal models showed that a tumor causes neutrophilia by upregulating granulocyte-colony stimulating factor (G-CSF) and granulocyte-macrophage-colony stimulating factor (GM-CSF) [14]. Neutrophils promote tumor growth through several mechanisms. One mechanism is angiogenesis by BV8 and vascular endothelial growth factor (VEGF) stimulated by neutrophils $[15,16]$. Another mechanism of pro-tumorigenic effects of neutrophils is inhibiting apoptosis [10]. Lastly, tumor-associated chronic inflammation leads to DNA damage by reactive oxygen species (ROS) or reactive nitrogen species (RNS) [17]. Platelet is also known to be associated with tumor growth, angiogenesis, and metastasis $[7,18]$.

Neutrophils and Iymphocytes participate in the regulation of adaptive immunity. Neutrophils express inducible nitric oxide synthase (iNOS) or arginase 1 (ARG1) and inhibit CD8+ T cell-mediated anti-tumor immunity [14]. Lymphopenia means the low number of $\mathrm{T}$ cell lymphocytes resulting in poor lymphocyte-mediated immune response to the tumor [3].

Previous studies have reported the use of NLR as a prognostic factor for NSCLC. Yin et al. [11] performed a metaanalysis of 14 studies with 2,734 cases to investigate the prognostic role of NLR in lung cancer. They demonstrated that elevated NLR showed worse OS in NSCLC $(H R=1.192 ; 95 \% \mathrm{Cl}$ 1.061-1.399) and SCLC (HR $=1.550 ; 95 \% \mathrm{Cl}, 1.061-2.077)$. This study includes all stages of NSCLC and different treatment methods.

The results of the stage III NSCLC study are as follows: Scilla et al. [10] reported that baseline NLR was a significant prognostic factor in patients with stage IIIA and IIIB NSCLC who received CCRT with or without surgery. Using cutoff point 5 , there was a difference in survival rate (median OS: 26 vs. 11 months; $p<0.0001)$. Tong et al. [3] also analyzed stage III NSCLC treated with CCRT or surgery followed by CRT. They found that patients with pre-treatment NLR $\geq 3.57$ had a significantly worse OS (5-year OS: 20\% vs. 8.8\%, p < 0.001). The abovementioned studies include some patients who underwent surgery as well as CCRT. In this study, we investigated the prognostic significance of the NLR in patients who received definitive CCRT and exclude treatment-related bias as less as possible.

A recent phase III trial added anti-programmed death ligand 1 (PD-L1) antibody durvalumab as consolidation therapy 
Table 2. Univariate analysis for OS and LRPFS

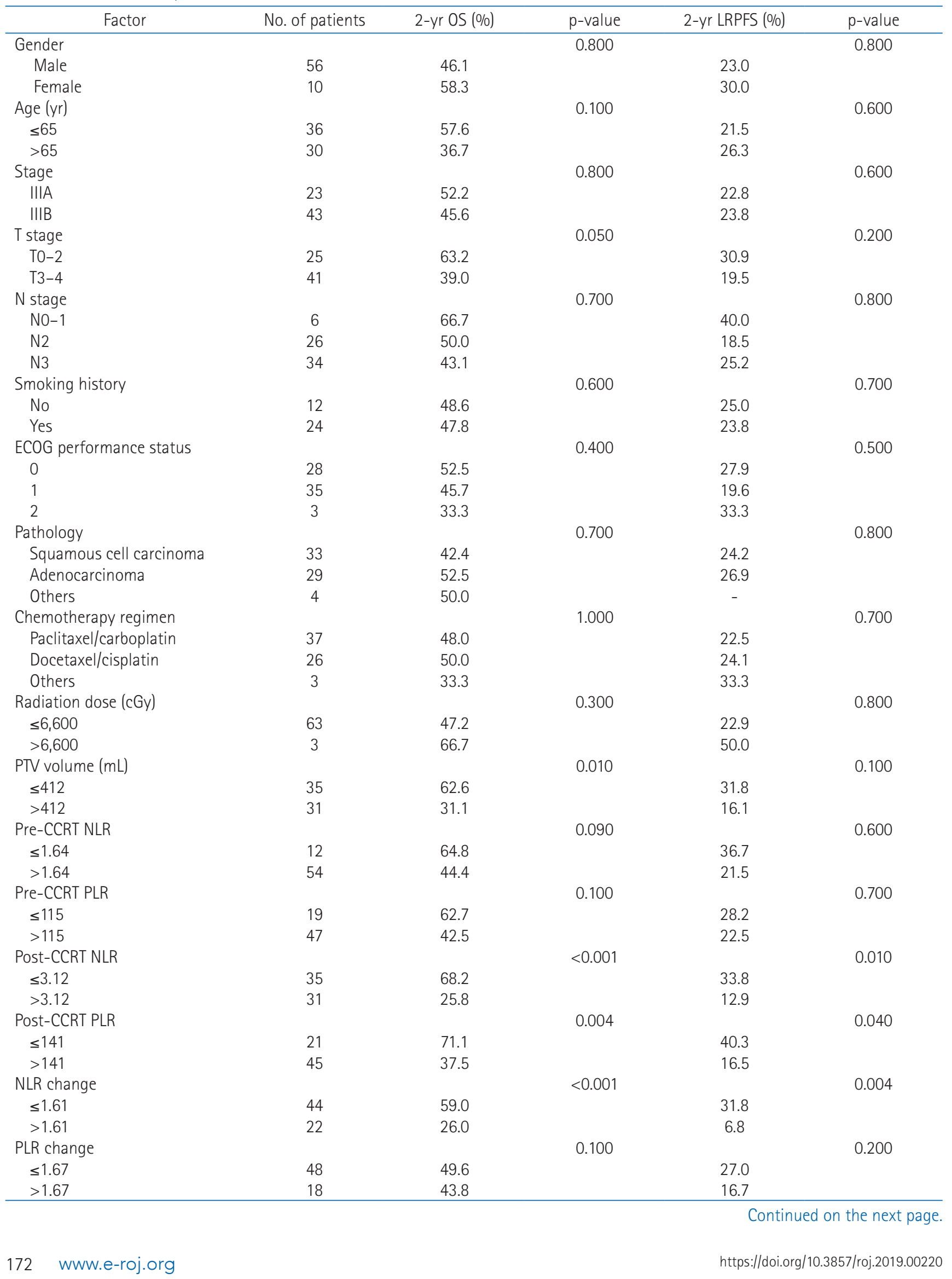


Table 2. Continued

\begin{tabular}{|c|c|c|c|c|c|}
\hline Factor & No. of patients & $2-y r$ OS (\%) & p-value & 2-yr LRPFS (\%) & $p$-value \\
\hline $\mathrm{CRP}(\mathrm{mg} / \mathrm{dL})$ & & & 0.090 & & 0.300 \\
\hline$\leq 0.47$ & 18 & 66.2 & & 25.9 & \\
\hline$>0.47$ & 45 & 40.0 & & 20.3 & \\
\hline ESR $(\mathrm{mm} / \mathrm{hr})$ & & & 0.200 & & 0.300 \\
\hline$\leq 15$ & 9 & 66.7 & & 33.3 & \\
\hline$>15$ & 54 & 44.3 & & 20.3 & \\
\hline LDH (U/L) & & & 0.700 & & 1.000 \\
\hline$\leq 450$ & 45 & 46.7 & & 24.1 & \\
\hline$>450$ & 21 & 51.3 & & 25.1 & \\
\hline
\end{tabular}

OS, overall survival; LRPFS, locoregional progression-free survival; ECOG, Eastern Cooperative Oncology Group; PTV, planning target volume; CCRT, concurrent chemoradiotherapy; NLR, neutrophil-to-lymphocyte ratio; PLR, platelet-to-lymphocyte ratio; NLR change, dividing the post-CCRT NLR by the pre-CCRT NLR; PLR change, dividing the post-CCRT PLR by the pre-CCRT PLR; CRP, C-reactive protein; ESR, erythrocyte sedimentation rate; LDH, lactate dehydrogenase.

Table 3. Multivariate analysis for OS and LRPFS

\begin{tabular}{|c|c|c|c|c|}
\hline \multirow{3}{*}{ PTV volume (mL) } & \multicolumn{2}{|c|}{ OS } & \multicolumn{2}{|c|}{ LRPFS } \\
\hline & $\mathrm{HR}(95 \% \mathrm{Cl})$ & \multirow[t]{2}{*}{ p-value } & $\mathrm{HR}(95 \% \mathrm{Cl})$ & \multirow[t]{2}{*}{$p$-value } \\
\hline & & & & \\
\hline$\leq 412$ & - & & - & \\
\hline$>412$ & $2.05(1.08-3.90)$ & 0.028 & 1.59 (0.89-2.86) & 0.116 \\
\hline \multicolumn{5}{|c|}{ Pre-CCRT NLR } \\
\hline$\leq 1.64$ & - & & - & \\
\hline$>1.64$ & $1.28(0.51-3.24)$ & 0.600 & $0.96(0.41-2.25)$ & 0.919 \\
\hline \multicolumn{5}{|c|}{ Pre-CCRT PLR } \\
\hline$\leq 115$ & - & & - & \\
\hline$>115$ & $0.78(0.35-1.76)$ & 0.553 & $0.71(0.32-1.54)$ & 0.385 \\
\hline \multicolumn{5}{|c|}{ Post-CCRT NLR } \\
\hline$\leq 3.12$ & - & & - & \\
\hline$>3.12$ & $1.60(0.68-3.81)$ & 0.285 & $1.56(0.70-3.52)$ & 0.279 \\
\hline \multicolumn{5}{|c|}{ Post-CCRT PLR } \\
\hline$\leq 141$ & - & & - & \\
\hline$>141$ & $2.18(0.89-5.33)$ & 0.088 & 1.79 (0.82-3.90) & 0.140 \\
\hline \multicolumn{5}{|c|}{ NLR change } \\
\hline$\leq 1.61$ & - & & - & \\
\hline$>1.61$ & 3.17 (1.16-8.69) & 0.025 & 1.71 (0.72-4.09) & 0.227 \\
\hline \multicolumn{5}{|c|}{ PLR change } \\
\hline$\leq 1.67$ & - & & - & \\
\hline$>1.67$ & $0.39(0.15-1.03)$ & 0.057 & 0.69 (0.31-1.54) & 0.361 \\
\hline
\end{tabular}

OS, overall survival; LRPFS, locoregional progression-free survival; $\mathrm{HR}$, hazard ratio; $\mathrm{Cl}$, confidence interval; PTV, planning target volume; CCRT, concurrent chemoradiotherapy; NLR, neutrophil-to-lymphocyte ratio; PLR, platelet-to-lymphocyte ratio; NLR change, dividing the post-CCRT NLR by the pre-CCRT NLR; PLR change, dividing the post-CCRT PLR by the pre-CCRT PLR.

for patients with stage III NSCLC who did not have disease progression after definitive CCRT [19]. Suh et al. [12] reported post-treatment NLR at week 6 was a prognostic marker in patients with advanced NSCLC treated with anti-PD-1 antibody. In a study by Khunger et al. [20], high post-treatment NLR after 2 cycles of nivolumab was associated with worse OS. In this study, post-CCRT NLR, post-CCRT PLR, and NLR change were significant prognostic factors for OS and LRPFS on the univariate analysis. However, age, stage, and ECOG performance status were not associated with survival. Even having the limitation of a retrospective study, the patients in our study had relatively homogeneous stage and ECOG performance status.

The PTV volume and NLR change were the significant 


\section{Eun Young Park, et al}

prognostic factors for OS in the multivariate analysis. Large tumor volume may indicate treatment resistance and a high probability of metastasis [21]. A previous study reported that radiation could affect circulating lymphocytes and cause lymphopenia [22]. In this study, PTV volume was larger in the high NLR change group than in the low NLR change group. It might be that the circulating neutrophils and lymphocytes were affected by the irradiated volume. However, there was no statistically significant correlation between PTV volume and absolute lymphocyte count after CCRT $(r=-0.138, p=0.270)$. Furthermore, there was no significant correlation between PTV volume and absolute neutrophil count after CCRT ( $r=0.144, p$ $=0.247$ ).

The results of the previous studies and this study showed that chronic inflammation plays a role in the resistance of chemoradiation treatment. NLR change between pre- and post-treatment are believed to indicate systemic inflammation and immune status reflecting the therapeutic effect. Despite high NLR before treatment, the patients whose NLR became low after treatment had better prognosis than the patients whose NLR remained high after treatment. Cannon et al. [23] suggested that PLR may be used in selecting adjuvant systemic treatment for early-stage lung cancer. We believe that patients with high NLR after treatment need frequent follow-up and aggressive adjuvant treatment.

Although NLR has been shown to be associated with patient survival, the cutoff point was so diverse. The range of cutoff points of NLR was from 2.5 to 5 according to a meta-analysis [6]. Our cutoff point for NLR change was 1.61. It is difficult to determine a reference point that can be clinically used; therefore, further studies are needed to confirm optimal cutoff values.

The limitations of our study were its retrospective singleinstitution design and small number of patients. Moreover, NLR could be affected by other conditions such as treatmentrelated toxicity. We used 4 weeks after treatment to exclude transient hematologic toxicity, but we cannot rule out that all effects are excluded. Thus, large multi-institutional studies are required to confirm the relationship between NLR and prognosis.

Therefore, we showed that NLR change was most significantly associated with OS in patients with locally advanced NSCLC treated with definitive CCRT. The elevated NLR after treatment may predict the patients at high risk of treatment failure.

\section{Conflict of Interest}

No potential conflict of interest relevant to this study was reported.

\section{References}

1. Ferlay J, Soerjomataram I, Dikshit $\mathrm{R}$, et al. Cancer incidence and mortality worldwide: sources, methods and major patterns in GLOBOCAN 2012. Int J Cancer 2015;136:E359-86.

2. Berghmans T, Paesmans M, Sculier JP. Prognostic factors in stage III non-small cell lung cancer: a review of conventional, metabolic and new biological variables. Ther Adv Med Oncol 2011;3:127-38.

3. Tong YS, Tan J, Zhou XL, Song YQ, Song YJ. Systemic immuneinflammation index predicting chemoradiation resistance and poor outcome in patients with stage III non-small cell lung cancer. J Transl Med 2017;15:221.

4. Vrankar M, Stanic K. Long-term survival of locally advanced stage III non-small cell lung cancer patients treated with chemoradiotherapy and perspectives for the treatment with immunotherapy. Radiol Oncol 2018;52:281-8.

5. National Comprehensive Cancer Network. NCCN Guidelines: non-small-cell lung cancer (version 2.2019) [Internet] Plymouth Meeting, PA: National Comprehensive Cancer Network; c2018 [cited 2019 Aug 21] Available from: https:// www.nccn.org/professionals/physician_gls/default.aspx.

6. Templeton AJ, McNamara MG, Seruga B, et al. Prognostic role of neutrophil-to-lymphocyte ratio in solid tumors: a systematic review and meta-analysis. J Natl Cancer Inst 2014;106:dju124.

7. Templeton AJ, Ace $0, M c N a m a r a ~ M G$, et al. Prognostic role of platelet to lymphocyte ratio in solid tumors: a systematic review and meta-analysis. Cancer Epidemiol Biomarkers Prev 2014;23:1204-12.

8. Son SH, Park EY, Park HH, Kay CS, Jang HS. Pre-radiotherapy neutrophil-to-lymphocyte ratio as an independent prognostic factor in patients with locally advanced hepatocellular carcinoma treated with radiotherapy. Oncotarget 2017:8:16964-71.

9. Sung S, Son SH, Park EY, Kay CS. Prognosis of locally advanced rectal cancer can be predicted more accurately using preand post-chemoradiotherapy neutrophil-lymphocyte ratios in patients who received preoperative chemoradiotherapy. PLoS One 2017;12:e0173955.

10. Scilla KA, Bentzen SM, Lam VK, et al. Neutrophil-lymphocyte ratio is a prognostic marker in patients with locally advanced 
(stage IIIA and IIIB) non-small cell lung cancer treated with combined modality therapy. Oncologist 2017;22:737-42.

11. Yin $Y$, Wang J, Wang $X$, et al. Prognostic value of the neutrophil to lymphocyte ratio in lung cancer: a metaanalysis. Clinics (Sao Paulo) 2015;70:524-30.

12. Suh KJ, Kim SH, Kim YJ, et al. Post-treatment neutrophil-tolymphocyte ratio at week 6 is prognostic in patients with advanced non-small cell lung cancers treated with anti-PD-1 antibody. Cancer Immunol Immunother 2018;67:459-70.

13. Kim TG, Park W, Choi DH, et al. Effect of leukocyte alteration on treatment outcomes following preoperative chemoradiotherapy in patients with rectal cancer. Radiat Oncol J 2017;35:217-26.

14. Coffelt SB, Wellenstein MD, de Visser KE. Neutrophils in cancer: neutral no more. Nat Rev Cancer 2016;16:431-46.

15. Nozawa $H$, Chiu C, Hanahan D. Infiltrating neutrophils mediate the initial angiogenic switch in a mouse model of multistage carcinogenesis. Proc Natl Acad Sci U S A 2006;103:12493-8.

16. Shojaei $F$, Wu $X$, Zhong $C$, et al. Bv8 regulates myeloid-celldependent tumour angiogenesis. Nature 2007;450:825-31.

17. Coussens LM, Werb Z. Inflammation and cancer. Nature 2002;420:860-7.

18. Jain S, Harris J, Ware J. Platelets: linking hemostasis and cancer. Arterioscler Thromb Vasc Biol 2010;30:2362-7.

19. Antonia SJ, Villegas $A$, Daniel D, et al. Durvalumab after chemoradiotherapy in stage III non-small-cell lung cancer. N Engl J Med 2017;377:1919-29.

20. Khunger M, Patil PD, Khunger $A$, et al. Post-treatment changes in hematological parameters predict response to nivolumab monotherapy in non-small cell lung cancer patients. PLoS One 2018;13:e0197743.

21. Liang SB, Teng JJ, Hu XF, et al. Prognostic value of total tumor volume in patients with nasopharyngeal carcinoma treated with intensity-modulated radiotherapy. BMC Cancer 2017;17:506.

22. Yovino $S$, Kleinberg $L$, Grossman SA, Narayanan M, Ford E. The etiology of treatment-related lymphopenia in patients with malignant gliomas: modeling radiation dose to circulating lymphocytes explains clinical observations and suggests methods of modifying the impact of radiation on immune cells. Cancer Invest 2013;31:140-4.

23. Cannon NA, Meyer J, lyengar $P$, et al. Neutrophil-lymphocyte and platelet-lymphocyte ratios as prognostic factors after stereotactic radiation therapy for early-stage non-small-cell lung cancer. J Thorac Oncol 2015;10:280-5. 